\title{
Minimum-Weight Edge Discriminators in Hypergraphs
}

\author{
Bhaswar B. Bhattacharya \\ Department of Statistics \\ Stanford University, California, U.S.A. \\ bhaswar@stanford.edu
}

\author{
Sayantan Das \\ Department of Biostatistics \\ University of Michigan, Ann Arbor, U.S.A. \\ sayantan@umich.edu
}

\author{
Shirshendu Ganguly \\ Department of Mathematics \\ University of Washington, Seattle, U.S.A. \\ sganguly@math. washington. edu
}

Submitted: Jul 7, 2013; Accepted: Jul 28, 2014; Published: Aug 6, 2014

Mathematics Subject Classifications: 05C65, 05C78, 05C38, 90C27

\begin{abstract}
In this paper we introduce the notion of minimum-weight edge-discriminators in hypergraphs, and study their various properties. For a hypergraph $\mathcal{H}=(\mathcal{V}, \mathscr{E})$, a function $\lambda: \mathcal{V} \rightarrow \mathbb{Z}^{+} \cup\{0\}$ is said to be an edge-discriminator on $\mathcal{H}$ if $\sum_{v \in E_{i}} \lambda(v)>0$, for all hyperedges $E_{i} \in \mathscr{E}$, and $\sum_{v \in E_{i}} \lambda(v) \neq \sum_{v \in E_{j}} \lambda(v)$, for every two distinct hyperedges $E_{i}, E_{j} \in \mathscr{E}$. An optimal edge-discriminator on $\mathcal{H}$, to be denoted by $\lambda_{\mathcal{H}}$, is an edge-discriminator on $\mathcal{H}$ satisfying $\sum_{v \in \mathcal{V}} \lambda_{\mathcal{H}}(v)=\min _{\lambda} \sum_{v \in \mathcal{V}} \lambda(v)$, where the minimum is taken over all edge-discriminators on $\mathcal{H}$. We prove that any hypergraph $\mathcal{H}=(\mathcal{V}, \mathscr{E})$, with $|\mathscr{E}|=m$, satisfies $\sum_{v \in \mathcal{V}} \lambda_{\mathcal{H}}(v) \leqslant m(m+1) / 2$, and the equality holds if and only if the elements of $\mathscr{E}$ are mutually disjoint. For $r$ uniform hypergraphs $\mathcal{H}=(\mathcal{V}, \mathscr{E})$, it follows from earlier results on Sidon sequences that $\sum_{v \in \mathcal{V}} \lambda_{\mathcal{H}}(v) \leqslant|\mathcal{V}|^{r+1}+o\left(|\mathcal{V}|^{r+1}\right)$, and the bound is attained up to a constant factor by the complete $r$-uniform hypergraph. Finally, we show that no optimal edge-discriminator on any hypergraph $\mathcal{H}=(\mathcal{V}, \mathscr{E})$, with $|\mathscr{E}|=m(\geqslant 3)$, satisfies $\sum_{v \in \mathcal{V}} \lambda_{\mathcal{H}}(v)=m(m+1) / 2-1$. This shows that all integer values between $m$ and $m(m+1) / 2$ cannot be the weight of an optimal edge-discriminator of a hypergraph, and this raises many other interesting combinatorial questions.
\end{abstract}

Keywords: Edge discrimination, Graph labeling, Hypergraphs, Irregular networks. 


\section{Introduction}

A hypergraph is a pair $\mathcal{H}=(\mathcal{V}, \mathscr{E})$ where $\mathcal{V}$ is a finite set and $\mathscr{E}$ is a collection of subsets of $\mathcal{V}$. The elements of $\mathcal{V}$ are called vertices and the elements of $\mathscr{E}$ are called hyperedges. A vertex labeling of a hypergraph is a function from the vertex set $\mathcal{V}$ to the set of nonnegative integers. In this paper, we introduce the notion of edge-discriminating vertex labelings in hypergraphs. A vertex labeling $\lambda: \mathcal{V} \rightarrow \mathbb{Z}^{+} \cup\{0\}$ is said to be an edgediscriminator on $\mathcal{H}$ if $\sum_{v \in E_{i}} \lambda(v)>0$, for all hyperedges $E_{i} \in \mathscr{E}$, and $\sum_{v \in E_{i}} \lambda(v) \neq$ $\sum_{v \in E_{j}} \lambda(v)$, for every two distinct hyperedges $E_{i}, E_{j} \in \mathscr{E}$. For any edge-discriminator $\lambda$ on $\mathcal{H}$, the value of the sum $\sum_{v \in \mathcal{V}} \lambda(v)$ will be called the weight of the edge-discriminator and shall be denoted by $\omega_{\lambda}(\mathcal{V})$. An edge-discriminator $\lambda_{\mathcal{H}}$ on $\mathcal{H}$ is said to be an optimal edge-discriminator if it has the least weight, that is, if $\omega_{\lambda_{\mathcal{H}}}(\mathcal{V})=\min _{\lambda} \omega_{\lambda}(\mathcal{V})$, where the minimum is taken over all edge-discriminators on $\mathcal{H}$. Henceforth, the weight of an optimal edge-discriminator on $\mathcal{H}$, that is, $\omega_{\lambda_{\mathcal{H}}}(\mathcal{V})$, will be denoted by $\omega_{0}(\mathcal{H})$.

\subsection{Related Works}

Graph labeling is an assignment of integers to the vertices or edges, or both, of a graph which satisfy certain conditions. Refer to the survey of Gallian [20] for a comprehensive view into the huge literature in graph labeling. Hypergraph vertex labelings where the sum of the labels of the vertices along the edges are mutually distinct, has been studied in the literature in many different contexts. One of them is the notion of anti-magic labeling of graphs. For a graph $G=(V, E)$ an edge-antimagic vertex labeling $l: V \rightarrow \mathbb{Z}^{+}$ is an injective function such that the quantities $l(x)+l(y)$ are mutually distinct, whenever $(x, y)$ is an edge in $G$. Edge-antimagic vertex labeling was studied by Wood [36]. Later, Bollobás and Pikhurko [6] defined the sum magic number of a graph $G=(V(G), E(G))$, denoted by $\mathcal{S}(G)$, as the smallest value of the largest vertex label in an edge-antimagic vertex labeling. They proved that $\mathcal{S}\left(K_{n}\right)=(1+o(1)) n^{2}$ and $\mathcal{S}(n, m)<(1-c) n^{2}$ whenever $m \leqslant c n^{2}$, where $\mathcal{S}(n, m)=\max \{\mathcal{S}(G):|V(G)|=n,|E(G)|=m\}$. Note that, unlike in the case of edge-discriminators, edge anti-magic vertex labeling of a graph is an injective function. Moreover, the sum magic number minimizes the maximum label, and not the the sum of the labels as required in the optimal edge-discriminator.

Another relevant topic is the notion of irregular networks. A network is a simple graph where each edge is assigned a positive integer weight. The degree of a vertex in a network is the sum of the weights of its incident edges. A network is irregular if all the vertices have distinct degrees. The strength of a network is the maximum weight assigned to any edge. The irregularity strength of a graph $G$ is the minimum strength among all irregular networks on $G$, and is denoted by $s(G)$. The notion of irregularity strength was first introduced by Chartrand et al. [8], where it was shown that for any given graph $G$,

$$
s(G) \geqslant \lambda(G)=\max _{i \leqslant j} \frac{\left(n_{i}+n_{i+1}+\ldots+n_{j}\right)+i-1}{j},
$$

where $n_{i}$ denotes the number of vertices of degree $i$. If $G$ contains a $K_{2}$ or multiple isolated vertices, the irregularity strength $s(G)=\infty$. Nierhoff [30] proved a tight bound 
$s(G) \leqslant n-1$, for graphs $G$ with $|V(G)|=n(>3)$ and $s(G)<\infty$. Faudree and Lehel [18] proved bounds on the irregularity strength of $d$-regular graphs. Upper bounds on the irregularity strength for general graphs, in terms of the minimum degree, were first given by Frieze et al. [19], and later by Przybyło [33, 34] and Kalkowski et al. [26]. However, computing the irregularity strength of a graph exactly is difficult in general. It is known only for some very special graphs and in almost all of these cases, it is found to be within an additive constant of $\lambda(G)$. It was conjectured by Lehel [27], that $s(G)$ is within an additive constant of $\lambda(G)$ for connected graphs. This has been verified for some special families of graphs like, complete graphs [8], cycles, most complete bipartite graphs, Turan graphs [17], wheels, hypercubes, and grids [13]. The notion of irregularity strength was extended to hypergraphs by Gyárfás et al. [23].

Edge-discriminators on hypergraphs and irregular networks are dual concepts. For a hypergraph $\mathcal{H}=(\mathcal{V}, \mathscr{E})$, the dual hypergraph is defined as $\mathcal{D}(\mathcal{H})=(\mathcal{W}, \mathcal{F})$, where $\mathcal{W}=\mathscr{E}$ and $\mathcal{F}=\{\mathscr{E}(v) \mid v \in \mathcal{V}\}$, where $\mathscr{E}(v)$ is the set of all edges in $\mathscr{E}$ which are incident on $v \in \mathcal{V}$. Note that if $\kappa: \mathscr{E} \rightarrow \mathbb{Z}^{+}$is an irregular edge assignment for $\mathcal{H}$, then $\kappa$ transforms to an edge-discriminator $\lambda: \mathcal{W} \rightarrow \mathbb{Z}^{+}$on $\mathcal{D}(\mathcal{H})$ as follows: For every vertex $v \in \mathcal{W}$, let $e_{v} \in \mathscr{E}$ be the corresponding hyperedge in $\mathcal{H}$ and define $\lambda(v)=\kappa\left(e_{v}\right)$. However, the most important difference between irregularity strength of a hypergraph and the optimal edge-discriminator in the dual hypergraph is the optimization criterion. In the case of irregularity strength the maximum label is minimized, whereas we minimize the sum of the labels in the optimal edge-discriminator. Another difference is that in an irregular network, the value assigned to an edge is always positive, which means that the edgediscriminator in the corresponding hypergraph assigns a positive weight to every vertex, which is not required by an edge-discriminator.

Another related problem involves the power set hypergraph. The power set hypergraph on a set $\mathcal{V}$, with $|\mathcal{V}|=n$, is the hypergraph $\left(\mathcal{V}, 2^{\mathcal{V}}\right)$, where $2^{\mathcal{V}}$ denotes the set of all nonempty subsets of $\mathcal{V}$. Note that any edge-discriminator on the power set hypergraph is a set of positive integers such that all its non-empty subsets have distinct sums. A set of positive numbers satisfying this property is called sum-distinct. A sum-distinct set of $m$ elements with the minimum total sum is the optimal edge-discriminator on the power set hypergraph, which can be easily computed to be $2^{|\mathcal{V}|}-1$. In 1931 Erdős asked for estimates of smallest possible value of the largest element in a sum-distinct set of $n$ elements, which we denote by $w(n)$. Erdös offered $\$ 500$ for verifying whether $w(n)=\Omega\left(2^{n}\right)$, and Guy [22] made the stronger conjecture that $w(n)>2^{n-3}$. In 1955 Erdős and Moser proved that $w(n) \geqslant 2^{n} /(4 \sqrt{n})[15]$. The constant was later improved by Elkies [14], which was further improved by Aliev [1]. A set consisting of the first $n$ powers of 2 has distinct subset sums, and has maximal element $2^{n-1}$, which implies that $w(n) \leqslant 2^{n-1}$. Conway and Guy [12] found a construction which gave an interesting upper bound on $w(n)$. This was later improved by Lunnon [29] and then by Bohman [5], who showed that $w(n)<0.22002 \cdot 2^{n}$ for sufficiently large $n$. 


\subsection{Our Results}

In this paper we study edge-discriminators on hypergraphs such that the sum of the labels of the vertices is minimized. We begin by proving a general upper bound on the weight of an edge-discriminator, which holds for any hypergraph. The bound is relatively simple to obtain, but it is tight.

Theorem 1. For any hypergraph $\mathcal{H}=(\mathcal{V}, \mathscr{E})$, with $|\mathscr{E}|=m, \omega_{0}(\mathcal{H}) \leqslant m(m+1) / 2$, and the equality holds if and only if the elements of $\mathscr{E}$ are mutually disjoint.

Next, we show that the edge-discrimination problem for $r$-uniform hypergraphs is related to Sidon sequences from additive number theory. A Sidon sequence is a sequence of natural numbers $A=\left\{a_{1}, a_{2}, \ldots\right\}$ such that all the pairwise sums $a_{i}+a_{j}(i \leqslant j)$ are different [16]. The $B_{h}$-sets are generalizations of Sidon-sequences in which all $h$-element sums are mutually distinct [31]. Using the connection between edge-discriminators and $B_{h}$ sets, we obtain another bound on the weight of the optimal edge-discriminator for $r$-uniform hypergraphs in terms of the number of vertices.

Proposition 2. For any r-uniform hypergraph $\mathcal{H}=(\mathcal{V}, \mathscr{E})$, with $|\mathcal{V}|=n, \omega_{0}(\mathcal{H}) \leqslant$ $n^{r+1}+o\left(n^{r+1}\right)$, and the bound is attained up to a constant factor by the optimal edgediscriminator of the complete $r$-uniform hypergraph on $\mathcal{V}$.

Obtaining nontrivial lower bounds on the weight of the optimal edge-discriminator for general hypergraphs seems to be difficult. It is easy to show that $\sum_{v \in \mathcal{V}} \lambda_{\mathcal{H}}(v) \geqslant$ $\max \{m, \delta(\delta+1) / 2\}$, where $|\mathscr{E}|=m$ and $\delta$ is the size of the maximum matching in $\mathcal{H}$. Moreover, there is a hypergraph, which attains this lower bound. However, like the irregularity strength, finding the optimal edge-discriminator is generally difficult for most hypergraphs. For examples where the optimal edge-discriminators can be explicitly computed refer to Bhattacharya et al. [2].

In Theorem 1 we show that the weight of an optimal edge-discriminator for a hypergraph with $m$ hyperedges is at most $m(m+1) / 2$. Moreover, the weight of any edgediscriminator is at least $m$. This motivates us to ask the following question: Given any integer $w \in[m, m(m+1) / 2]$, whether there exists a hypergraph $\mathcal{H}$ with $m$ hyperedges such that $w$ is the weight of the optimal edge-discriminator on $\mathcal{H}$. We provide a negative answer, by proving the following theorem:

Theorem 3. There exists no hypergraph $\mathcal{H}=(\mathcal{V}, \mathscr{E})$ with $|\mathscr{E}|=m(\geqslant 3)$, such that the weight of the optimal edge-discriminator on $\mathcal{H}$ is $m(m+1) / 2-1$.

The above result indicates that the problem of attainability of weights is an interesting combinatorial problem which might have surprising consequences. We discuss the attainability problem in more detail later on.

The rest of the paper is organized as follows: The proof of Theoerem 1 where we give a general upper bound on the weight of an optimal edge-discriminator is given in Section 2 . In Section 3 we outline the connection between Sidon sequences and edge-discriminators in uniform hypergraphs. A short discussion on lower bounds is given in Section 4. The 
problem of non-attainable weights and the proof of Theorem 3 are in Section 5. In Section 6 we discuss edge-discriminators in geometric hypergraphs and its potential application to digital image indexing. Finally, in Section 7 we summarize our work and give directions for future research.

\section{Constructing Edge-Discriminators in General Hy- pergraphs}

In this section, we give an algorithm for constructing an edge-discriminator for a general hypergraph. We begin by introducing some definitions and notations. For any set $S$, denote by $|S|$ the cardinality of $S$. Consider a hypergraph $\mathcal{H}=(\mathcal{V}, \mathscr{E})$, with $|\mathcal{V}|=n$ and $\mathscr{E}=\left\{E_{1}, E_{2}, \ldots, E_{m}\right\}$, where $|\mathscr{E}|=m$ and $E_{i} \subseteq \mathcal{V}$ for $i \in[m]:=\{1,2, \ldots, m\}$. An ordering on $\mathcal{V}$ is a bijective function $\nu:[n] \rightarrow \mathcal{V}$. We shall write $\nu_{i}:=\nu(i)$, for $i \in[n]$. Thus, with respect to the ordering $\nu$, the vertices in $\mathcal{V}$ will be indexed as $\left\{\nu_{1}, \nu_{2}, \ldots, \nu_{n}\right\}$. For two vertices $\nu_{i}, \nu_{j} \in \mathcal{V}$, we say $\nu_{i}$ is less than $\nu_{j}$ with respect to $\nu$, if $i<j$. The maximal vertex of $\mathcal{W} \subset \mathcal{V}$ is the vertex $\nu_{k} \in \mathcal{W}$ such that for all vertices $\nu_{i} \in \mathcal{W} \backslash\left\{\nu_{k}\right\}$, we have $i<k$. The maximal vertex of $\mathcal{W}$ will be denoted by $\nu(\mathcal{W})$. For two hyperedges $E_{i}, E_{j} \in \mathscr{E}(i \neq j)$ the vertex $\nu\left(E_{i}, E_{j}\right):=\nu\left(E_{i} \Delta E_{j}\right)$ will be called the differentiating vertex of the edges $E_{i}$ and $E_{j}$, where for any two sets $A$ and $B, A \Delta B=(A \backslash B) \cup(B \backslash A)$. The edge which contains the differentiating vertex $\nu\left(E_{i}, E_{j}\right)$ will be denoted by $\bar{E}_{i j}$, and the edge which does not contain the differentiating vertex will be denoted by $\underline{E}_{i j}$.

For any function $\lambda: \mathcal{V} \rightarrow \mathbb{Z}^{+} \cup\{0\}$, the weight of any subset $\mathcal{W}$ of $\mathcal{V}$ is defined as $\omega_{\lambda}(\mathcal{W})=\sum_{v \in \mathcal{W}} \lambda(v)$. Thus, a function $\lambda$ is edge-discriminating if the weights of all the edges in $\mathscr{E}$ are distinct.

\subsection{Proof of Theorem 1}

Given a hypergraph $\mathcal{H}=(\mathcal{V}, \mathscr{E})$, with $|\mathcal{V}|=n$ and $|\mathscr{E}|=m$, consider the hypergraph $\mathcal{H}_{0}=$ $(\mathcal{V}, \mathcal{F})$, where $\mathcal{F}=\mathscr{E} \cup\{\emptyset\}$. Fix an ordering $\nu$ on $\mathcal{V}$. Let $\lambda: \mathcal{V} \rightarrow \mathbb{Z}^{+} \cup\{0\}$ be a function initialized as $\lambda(v)=0$, for all $v \in \mathcal{V}$. We iteratively update the value of the function at the vertices according to the ordering induced by $\nu$. Abusing notation we will denote the function by $\lambda$ throughout the iterative procedure. Once $\lambda\left(\nu_{1}\right), \lambda\left(\nu_{2}\right), \ldots, \lambda\left(\nu_{k-1}\right)$ are updated, update $\lambda\left(\nu_{k}\right)$ by adding the least non-negative integer not in the set

$$
\mathcal{A}\left(\nu_{k}\right)=\left\{\omega_{\lambda}\left(\underline{E}_{i j}\right)-\omega_{\lambda}\left(\bar{E}_{i j}\right): \nu\left(E_{i}, E_{j}\right)=\nu_{k}, E_{i}, E_{j} \in \mathcal{F}\right\} .
$$

This implies that $\lambda\left(\nu_{k}\right)$ is at most $\left|\mathcal{A}\left(\nu_{k}\right)\right|$, since initially $\lambda\left(\nu_{k}\right)$ was 0 . Note that for any two hyperedges $E_{i}, E_{j} \in \mathcal{F}$, such that $\nu\left(E_{i}, E_{j}\right)=\nu_{k}$, all the vertices which are greater than $\nu_{k}$ are common to both or belong to neither of the edges $E_{i}$ and $E_{j}$. Therefore, according to the above construction, $\omega_{\lambda}\left(\underline{E}_{i j}\right)-\omega_{\lambda}\left(\bar{E}_{i j}\right)$ cannot change once $\lambda\left(\nu_{k}\right)$ is assigned. Hence, by the choice of $\lambda\left(\nu_{k}\right), \omega_{\lambda}\left(E_{i}\right) \neq \omega_{\lambda}\left(E_{j}\right)$, from the $k$-th step onwards. In particular, taking one of the edges in the pair as $\emptyset$ implies that $\omega_{\lambda}\left(E_{i}\right)>0$ for all $E_{i} \in \mathscr{E}$, as $\omega_{\lambda}(\emptyset)=0$. 
Therefore, at the end of the updating procedure, the function $\lambda$ is an edge-discriminator on $\mathcal{H}=(\mathcal{V}, \mathscr{E})$.

Next, observe that $\left|\mathcal{A}\left(\nu_{k}\right)\right|$ is at most the number of pairs of edges in $\mathcal{F}$ which has $\nu_{k}$ as a differentiating vertex. As $\nu\left(E_{i}, \emptyset\right)=\nu\left(E_{i}\right)$, it immediately follows that $\left|\mathcal{A}\left(\nu_{k}\right)\right| \leqslant$ $\chi\left(\nu_{k}\right)+\pi\left(\nu_{k}\right)$, where $\chi\left(\nu_{k}\right)$ denotes the number of pairs of edges in $\mathscr{E}$ for which $\nu_{k}$ is a differentiating vertex, and $\pi\left(\nu_{k}\right)$ is the number of edges in $\mathscr{E}$ for which $\nu_{k}$ is the maximal vertex. This implies that

$$
\begin{aligned}
\sum_{v \in \mathcal{V}} \lambda(v)=\sum_{k=1}^{|\mathcal{V}|} \lambda\left(\nu_{k}\right) & \leqslant \sum_{k=1}^{n} \chi\left(\nu_{k}\right)+\sum_{k=1}^{n} \pi\left(\nu_{k}\right) \\
& =\frac{m(m-1)}{2}+m=\frac{m(m+1)}{2}
\end{aligned}
$$

which completes the proof of the first part of Theorem 1.

\subsubsection{General Algorithm for Constructing Edge-Discriminators}

Before completing the proof of Theorem 1, we slightly generalize our procedure for constructing an edge-discriminator. This will be needed to complete the proof of Theorem 1. Moreover, it gives better insight into the structure of the discriminating functions, and ultimately leads to an improved upper bound on the weight of the optimal edgediscriminator.

The general algorithm is very similar to the algorithm in the proof of Theorem 1 , but instead of starting with the zero function, we start with any arbitrary integer-valued function $\kappa$, and execute the same procedure to get an edge-discriminator. Typically, depending on the structure of the hypergraph one can choose the initial function to obtain sharper bounds. More formally, the Construction Algorithm takes a hypergraph $\mathcal{H}=(\mathcal{V}, \mathscr{E})$, an ordering $\nu$ on $\mathcal{V}$, and any function $\kappa: \mathcal{V} \rightarrow \mathbb{Z}^{+} \cup\{0\}$, and returns an edge-discriminator $\lambda_{\kappa, \nu}: \mathcal{V} \rightarrow \mathbb{Z}^{+} \cup\{0\}$.

Lemma 1. Given any hypergraph $\mathcal{H}=(\mathcal{V}, \mathscr{E})$, an ordering $\nu$ on $\mathcal{V}$, and any function $\kappa: \mathcal{V} \rightarrow \mathbb{Z}^{+} \cup\{0\}$, the Construction Algorithm produces an edge-discriminator $\lambda_{\kappa, \nu}: \mathcal{V} \rightarrow$ $\mathbb{Z}^{+} \cup\{0\}$ on $\mathcal{H}$ such that $\omega_{\lambda_{\kappa, \nu}}(\mathcal{V}) \leqslant \frac{m(m+1)}{2}-\sum_{k=1}^{n} \pi\left(\nu_{k}\right) \mathbf{1}\left\{\kappa\left(\nu_{k}\right)>0\right\}+\sum_{k=1}^{n} \kappa\left(\nu_{k}\right)$.

Proof. The proof that $\lambda_{\kappa, \nu}$ is an edge-discriminator is very similar to the proof of Theorem 1. For $\kappa\left(\nu_{k}\right)=0$, we compare all pair of hyperedges $E_{i}, E_{j} \in \mathcal{F}$, such that $\nu\left(E_{i}, E_{j}\right)=\nu_{k}$. As all the vertices which are greater than $\nu_{k}$ are common to both or belong to neither of the edges $E_{i}$ and $E_{j}$, according to the construction $\omega_{\lambda}\left(\underline{E}_{i j}\right)-\omega_{\lambda}\left(\bar{E}_{i j}\right)$ cannot change once $\lambda\left(\nu_{k}\right)$ is assigned. Hence, by the choice of $\lambda\left(\nu_{k}\right), \omega_{\lambda}\left(E_{i}\right) \neq \omega_{\lambda}\left(E_{j}\right)$, from the $k$-th step onwards and therefore eventually, for any pair of edges such that $\nu\left(E_{i}, E_{j}\right)=\nu_{k}$ and $\kappa\left(\nu_{k}\right)=0$.

When $\kappa\left(\nu_{k}\right)>0$, we define $\lambda_{\kappa, \nu}\left(\nu_{k}\right)=\kappa\left(\nu_{k}\right)+\inf \left\{\mathbb{Z}^{+} \cup\{0\} \backslash \mathcal{B}\left(\nu_{k}\right)\right\}$. This choice of $\lambda\left(\nu_{k}\right)$ differentiates any pair of edges $E_{x}, E_{y} \in \mathscr{E}$, with $\nu\left(E_{x}, E_{y}\right)=\nu_{k}$. Note that here we ignore the pairs $\left(E_{z}, \emptyset\right)$, where $\nu\left(E_{z}, \emptyset\right)=\nu_{k}$. However, as $\kappa\left(\nu_{k}\right)>0, \omega_{\lambda_{\kappa, \nu}}\left(E_{z}\right)>0$, 
Input: A hypergraph $\mathcal{H}=(\mathcal{V}, \mathscr{E})$, an ordering $\nu$ on $\mathcal{V}$, and any function $\kappa: \mathcal{V} \rightarrow \mathbb{Z}^{+} \cup\{0\}$.

Output: An edge-discriminator $\lambda_{\kappa, \nu}: \mathcal{V} \rightarrow \mathbb{Z}^{+} \cup\{0\}$.

Initialize $\lambda_{\kappa, \nu}(v)=\kappa(v)$, for all $v \in \mathcal{V}$.

while $1<k \leqslant n$ do

Denote $\mathcal{B}\left(\nu_{k}\right)=\left\{\left|\omega_{\lambda_{\kappa, \nu}}\left(\underline{E}_{i j}\right)-\omega_{\lambda_{\kappa, \nu}}\left(\bar{E}_{i j}\right)\right|: \nu\left(E_{i}, E_{j}\right)=\nu_{k}, E_{i}, E_{j} \in \mathscr{E}\right\}$, $\mathcal{C}\left(\nu_{k}\right)=\left\{\omega_{\lambda_{\kappa, \nu}}\left(E_{i}\right): \nu\left(E_{i}, \emptyset\right)=\nu_{k}, E_{i} \in \mathscr{E}\right\}$, and $\mathcal{A}\left(\nu_{k}\right)=\mathcal{B}\left(\nu_{k}\right) \cup \mathcal{C}\left(\nu_{k}\right)$;

if $\left|\mathcal{A}\left(\nu_{k}\right)\right| \neq 0$ then

$$
\text { if } \kappa\left(\nu_{k}\right)=0 \text { then }
$$

Define $\lambda_{\kappa, \nu}\left(\nu_{k}\right)=\inf \left\{\mathbb{Z}^{+} \cup\{0\} \backslash \mathcal{A}\left(\nu_{k}\right)\right\}$,

\section{end}

if $\kappa\left(\nu_{k}\right)>0$ then

Define $\lambda_{\kappa, \nu}\left(\nu_{k}\right)=\kappa\left(\nu_{k}\right)+\inf \left\{\mathbb{Z}^{+} \cup\{0\} \backslash \mathcal{B}\left(\nu_{k}\right)\right\}$.

end

else

| Define $\lambda_{\kappa, \nu}\left(\nu_{k}\right)=\kappa\left(\nu_{k}\right)$,

end

end

$k \leftarrow k+1$.

Return the function $\lambda_{\kappa, \nu}: \mathcal{V} \rightarrow \mathbb{Z}^{+} \cup\{0\}$.

Algorithm 1: Construction Algorithm: Edge-Discriminator Construction Algorithm.

for any edge $E_{z}$ such that $\nu\left(E_{z}, \emptyset\right)=\nu_{k}$. Hence, any pair of edges has distinct weights in this case as well.

Now, from the algorithm we can bound the weight of $\lambda_{\kappa, \nu}$ as follows:

$$
\begin{aligned}
\sum_{k=1}^{n} \lambda_{\kappa, \nu}\left(\nu_{k}\right) & \leqslant \sum_{k=1}^{n}\left|\mathcal{A}\left(\nu_{k}\right)\right| \mathbf{1}\left\{\kappa\left(\nu_{k}\right)=0\right\}+\sum_{k=1}^{n}\left|\mathcal{B}\left(\nu_{k}\right)\right| \mathbf{1}\left\{\kappa\left(\nu_{k}\right)>0\right\}+\sum_{k=1}^{n} \kappa\left(\nu_{k}\right) \\
& \leqslant \sum_{k=1}^{n}\left|\mathcal{B}\left(\nu_{k}\right)\right|+\sum_{k=1}^{n}\left|\mathcal{C}\left(\nu_{k}\right)\right| \mathbf{1}\left\{\kappa\left(\nu_{k}\right)=0\right\}+\sum_{k=1}^{n} \kappa\left(\nu_{k}\right) \\
& \leqslant \sum_{k=1}^{n} \chi\left(\nu_{k}\right)+\sum_{k=1}^{n} \pi\left(\nu_{k}\right) \mathbf{1}\left\{\kappa\left(\nu_{k}\right)=0\right\}+\sum_{k=1}^{n} \kappa\left(\nu_{k}\right) \\
& =\frac{m(m-1)}{2}+\sum_{k=1}^{n} \pi\left(\nu_{k}\right)-\sum_{k=1}^{n} \pi\left(\nu_{k}\right) \mathbf{1}\left\{\kappa\left(\nu_{k}\right)>0\right\}+\sum_{k=1}^{n} \kappa\left(\nu_{k}\right) \\
& =\frac{m(m+1)}{2}-\sum_{k=1}^{n} \pi\left(\nu_{k}\right) \mathbf{1}\left\{\kappa\left(\nu_{k}\right)>0\right\}+\sum_{k=1}^{n} \kappa\left(\nu_{k}\right) .
\end{aligned}
$$

We will use the above lemma in completing the proof of Theorem 1 and also in the proof of Theorem 3 . 


\subsubsection{Completing the Proof of Theorem 1}

To complete the proof of Theorem 1 we need to show that given any hypergraph $\mathcal{H}=$ $(\mathcal{V}, \mathscr{E})$, which contains two egdes $E_{x}, E_{y} \in \mathscr{E}$ such that $E_{x} \cap E_{y} \neq \emptyset$, it is possible to construct an edge-discriminating function $\lambda$ on $\mathcal{V}$ such that $\sum_{v \in \mathcal{V}} \lambda(v)<m(m+1) / 2$.

Let $|\mathcal{V}|=n$ and $v_{o} \in E_{x} \cap E_{y}$. Consider an ordering $\widetilde{\nu}:[n] \rightarrow \mathcal{V}$ such that $\widetilde{\nu}_{n}=\widetilde{\nu}(n)=$ $v_{o}$. Then $v_{o}$ is the maximal vertex of both the edges $E_{x}$ and $E_{y}$, that is, $\pi\left(\widetilde{\nu}_{n}\right) \geqslant 2$. We start with the function $\kappa=\delta_{v_{o}}$ which takes the value 1 at $v_{o}$ and 0 everywhere else. Then from Lemma 1, we know that there exists an edge-discriminator $\lambda_{\kappa, \widetilde{\nu}}$ such that $\sum_{v \in \mathcal{V}} \lambda_{\kappa, \widetilde{\nu}}(v) \leqslant m(m+1) / 2-1$, which proves the tightness of the upper bound and completes the proof of Theorem 1 .

\subsubsection{Consequences of Lemma 1}

In this section we discuss an immediate corollary of Lemma 1 which gives a slightly better upper bound on the weight of an edge-discriminator than in Theorem 1.

To this end, we need the following definition. For a hypergraph $\mathcal{H}=(\mathcal{V}, \mathscr{E})$, a set $S \subseteq \mathcal{V}$ is called a hitting set of $\mathcal{H}$ if, for all edges $E \in \mathscr{E}, S \cap E \neq \emptyset$. A hitting set of the smallest size is called the minimum hitting set of $\mathcal{H}$. Hitting sets are also known as vertex covers or transversals.

Corollary 4. For any hypergraph $\mathcal{H}=(\mathcal{V}, \mathscr{E}), \omega_{0}(\mathcal{H}) \leqslant \frac{m(m-1)}{2}+\mathcal{N}(\mathcal{H})$, where $\mathcal{N}(\mathcal{H})$ is the size of the minimum hitting set of $\mathcal{H}$.

Proof. For a fixed ordering $\nu$, denote by $N(\nu)$ the set of vertices $v \in \mathcal{V}$ with $\pi(v)>0$. Observe that $\min _{\nu}|N(\nu)|=\mathcal{N}(\mathcal{H})$.

Now, consider any ordering $\nu$ on $\mathcal{V}$. Define $\kappa(v)=\mathbf{1}\{\pi(v)>0\}$. Lemma 1 then implies that there exists an edge-discriminator $\lambda_{\kappa, \nu}$ such that $\sum_{v \in \mathcal{V}} \lambda_{\kappa, \nu}(v) \leqslant m(m-1) / 2+|N(\nu)|$. The result follows by taking minimum over all orderings on $\mathcal{V}$.

Remark 1. Note that the bound in Corollary 4 is slightly better than the general upper bound proved in Theorem 1. Moreover, it is easy to see that this bound is attained by the optimal edge-discriminator of the star-graph $T_{m}$ on $m+1$ vertices and $m$ edges. This follows from the fact that the central vertex of $T_{m}$ is the minimum hitting set for $T_{m}$, and so $\mathcal{N}(\mathcal{H})=1$.

\section{Edge Discriminators in $r$-Uniform Hypergraphs}

A $r$-uniform hypergraph is a hypergraph $\mathcal{H}=(\mathcal{V}, \mathscr{E})$, where all the hyperedges in $\mathscr{E}$ have cardinality $r$. If $\mathscr{E}$ consists of the set of all $r$-element subsets of $\mathcal{V}$ then the hypergraph $\mathcal{H}=(\mathcal{V}, \mathscr{E})$ is called the complete $r$-uniform hypergraph on $\mathcal{V}$, and is denoted by $\mathcal{K}_{n}^{r}$.

Consider a $r$-uniform hypergraph on $n$-vertices and let $\mathcal{V}=\left\{v_{1}, v_{2}, \ldots, v_{n}\right\}$. We can obtain an edge discriminator $\lambda: \mathcal{V} \rightarrow \mathbb{Z}^{+} \cup\{0\}$ if the sequence $\left\{\lambda\left(v_{1}\right), \lambda\left(v_{2}\right), \ldots, \lambda\left(v_{n}\right)\right\}$, has the property that the sum of the elements in each its $r$-element subset is distinct. This property of the sequence $\lambda\left(v_{1}\right), \lambda\left(v_{2}\right), \ldots, \lambda\left(v_{n}\right)$ is closely related to the notion of Sidon sequences from additive number theory. 


\subsection{Sidon Sequences and Generalizations}

A Sidon sequence is a finite or infinite sequence of natural numbers $A=\left\{a_{1}, a_{2}, \ldots\right\}$ such that all the pairwise sums $a_{i}+a_{j}(i \leqslant j)$ are different. This problem was introduced by Sidon in 1932, during his investigations in Fourier analysis. A celebrated combinatorial problem asks for estimates of the maximum number of elements $s(N)$ from $\{1,2, \ldots, N\}$ which form a Sidon sequence. This was posed by Erdös and Turán [16], and they proved that $s(N) \leqslant N^{1 / 2}+O\left(N^{1 / 4}\right)$, which is the best possible upper bound except for the estimate of the error-term. The upper bound was refined by Lindström [28] to $s(N) \leqslant$ $N^{1 / 2}+N^{1 / 4}+1$ and further improved by Cilleruello [9]. A conjecture of Erdős, with a $\$ 500$ prize, says that $s(N)=N^{1 / 2}+O(1)$.

Sidon-sequences can be generalized by considering sequences in which all $h$-element sums are mutually distinct. This leads to the following definition:

Definition 5. For a positive integer $h \geqslant 2$, a finite or infinite sequence of positive integers $A=\left\{a_{1}, a_{2}, \ldots\right\}$ is called a $B_{h}$-set if for every positive integer $c$, the equation

$$
c=a_{1}+a_{2}+\ldots+a_{h}, \quad a_{1} \leqslant a_{2} \leqslant \ldots, \leqslant a_{h}, \quad a_{i} \in A,
$$

has, at most, one solution. Let $F_{h}(N)$ denote the cardinality of the largest $B_{h}$ set that can be selected from the set $\{1,2, \ldots, N\}$.

Bose and Chowla [7] proved that $F_{h}(N) \geqslant N^{1 / h}+o\left(N^{1 / h}\right)$. An easy counting argument shows $F_{h}(N) \leqslant(h h ! N)^{1 / h}$. This upper bound has gone through many improvements and refinements. The general upper bound has the form $F_{h}(N) \leqslant c(h) N^{1 / h}+o\left(N^{1 / h}\right)$, where $c(h)$ is a constant depending on $h$. For specific values of $c(h)$ refer to Cilleruello [10], Cilleruello and Jimenez [11], Jia [25], and the references therein. Other related results and problems on Sidon sequences and $B_{h}$-sets can be found in the surveys $[24,31]$.

\subsection{Proof of Proposition 2}

In this section we use the notion of $B_{h}$-sets to obtain new bounds on the weight of an edge-discriminator in $r$-uniform hypergraphs. Consider a $r$-uniform hypergraph $\mathcal{H}=$ $(\mathcal{V}, \mathscr{E})$, with $\mathcal{V}=\left\{v_{1}, v_{2}, \ldots, v_{n}\right\}$. Note that a function $\lambda: \mathcal{V} \rightarrow \mathbb{Z}^{+} \cup\{0\}$ such that $\left\{\lambda\left(v_{1}\right), \lambda\left(v_{2}\right), \ldots, \lambda\left(v_{n}\right)\right\}$ is a $B_{r}$-set, is an edge-discriminator for $\mathcal{H}$. Let $G_{h}(n)$ be the minimum of the maximum element taken over all $B_{h}$-sets of length $m$. In other words, $G_{h}(n)$ is the inverse function of $F_{h}(n)$, and a lower bound for $F_{h}(n)$ corresponds to an upper bound for $G_{h}(n)$. Now, as $G_{r}(n) \leqslant n^{r}+o\left(n^{r}\right)$, we have

$$
\omega_{\lambda}(\mathcal{H})=\sum_{i=1}^{n} \lambda\left(v_{i}\right) \leqslant n \cdot \max _{1 \leqslant i \leqslant n} \lambda\left(v_{i}\right) \leqslant n^{r+1}+o\left(n^{r+1}\right) .
$$

In the case of the complete $r$-uniform hypergraph $\mathcal{K}_{n}^{r}=(\mathcal{V}, \mathscr{E}),|\mathscr{E}|=\left(\begin{array}{l}n \\ r\end{array}\right)$ and every vertex $v \in \mathcal{V}$ belongs to $\left(\begin{array}{l}n-1 \\ r-1\end{array}\right)$ hyperedges. This implies that any edge-discriminator 
$\lambda: \mathcal{V} \rightarrow \mathbb{Z}^{+} \cup\{0\}$ on $\mathcal{K}_{n}^{r}$ satisfies

$$
\sum_{E \in \mathscr{E}} \omega_{\lambda}(E)=\sum_{E \in \mathscr{E}} \sum_{v \in E} \lambda(v)=\left(\begin{array}{c}
n-1 \\
r-1
\end{array}\right) \sum_{v \in \mathcal{V}} \lambda(v) .
$$

As $\omega_{\lambda}(E)$ is distinct for all $E \in \mathscr{E}, \sum_{E \in \mathscr{E}} \omega_{\lambda}(E) \geqslant|\mathscr{E}|(|\mathscr{E}|+1) / 2$. Therefore,

$$
\omega_{\lambda}\left(\mathcal{K}_{n}^{r}\right)=\sum_{v \in \mathcal{V}} \lambda(v)=\frac{1}{\left(\begin{array}{l}
n-1 \\
r-1
\end{array}\right)} \sum_{E \in \mathscr{E}} \omega_{\lambda}(E) \geqslant \frac{\left.\left(\begin{array}{l}
n \\
r
\end{array}\right)\left(\begin{array}{l}
n \\
r
\end{array}\right)+1\right)}{2\left(\begin{array}{l}
n-1 \\
r-1
\end{array}\right)}=\frac{\frac{n}{r}\left(\left(\begin{array}{l}
n \\
r
\end{array}\right)+1\right)}{2} \geqslant c n^{r+1},
$$

for $n$ large enough and some constant $c$. This proves that the weight of the optimal edge-discriminator of $\mathcal{K}_{n}^{r}$ is within a constant factor of the upper bound, and completes the proof of Proposition 2.

Remark 2. The upper bound on the weight of an edge-discriminator for a $r$-uniform hypergraph obtained in Proposition 2 is often better than the general $|\mathscr{E}|(|\mathscr{E}|+1) / 2=$ $O\left(|\mathscr{E}|^{2}\right)$ upper bound proved in Theorem 1. In particular, when $|\mathscr{E}| \geqslant c|\mathcal{V}|^{\frac{r+1}{2}}$, then Proposition 2 provides a better bound on the weight of an edge-discriminator. For example, if the $r$-uniform hypergraph is dense then Proposition 2 gives a sharper upper bound. On the other hand, if we have a sparse $r$-uniform hypergraph, then Theorem 1 gives a better bound.

\section{Lower Bound}

In this section we prove a simple lower bound on the weight of an edge discriminator on $\mathcal{H}$. Given a hypergraph $\mathcal{H}=(\mathcal{V}, \mathscr{E})$, a subset $\mathscr{E} \subseteq \mathscr{E}$ is said to be a matching if the elements in $\mathscr{E}^{\prime}$ are mutually disjoint. A maximum matching of $\mathcal{H}$ is the largest size matching in $\mathcal{H}$.

Proposition 6. For any edge-discriminator $\lambda$ on a hypergraph $\mathcal{H}=(\mathcal{V}, \mathscr{E})$, with $|\mathscr{E}|=m$, $\omega_{\lambda}(\mathcal{V}) \geqslant \max \left\{m, \frac{\delta(\delta+1)}{2}\right\}$, where $\delta$ is the size of the maximum matching of $\mathcal{H}$. Moreover, there is a hypergraph with $m$ edges which attains this bound.

Proof. Observe that the weights $\omega_{\lambda}\left(E_{1}\right), \omega_{\lambda}\left(E_{2}\right), \ldots, \omega_{\lambda}\left(E_{m}\right)$ are all positive and distinct. This implies that

$$
\omega_{\lambda}(\mathcal{V}) \geqslant \max _{E_{i} \in \mathscr{E}} \omega_{\lambda}\left(E_{i}\right) \geqslant m
$$

Next, suppose that $\mathscr{E}^{\prime}=\left\{E_{i_{1}}, E_{i_{2}}, \ldots, E_{i_{\delta}}\right\}$ is a matching of $\mathcal{H}$. Since the hyperedges $E_{i_{1}}, E_{i_{2}}, \ldots, E_{i_{\delta}}$ are mutually disjoint and the corresponding weights $\omega_{\lambda}\left(E_{i_{1}}\right)$, $\omega_{\lambda}\left(E_{i_{2}}\right), \ldots, \omega_{\lambda}\left(E_{i_{\delta}}\right)$ are distinct,

$$
\omega_{\lambda}(\mathcal{V}) \geqslant \sum_{j=1}^{\delta} \omega_{\lambda}\left(E_{i_{j}}\right) \geqslant \frac{\delta(\delta+1)}{2} .
$$

Finally, consider the hypergraph $\mathcal{H}_{1}=\left(\mathcal{V}_{1}, \mathscr{E}_{1}\right)$, with $\mathcal{V}_{1}=[n]$ and $\mathscr{E}_{1}=\left\{E_{1}\right.$, $\left.E_{2}, \ldots, E_{m}\right\}$, where $E_{i}=[i]$. Then it is easy to see that the function $\lambda_{1}: \mathcal{V}_{1} \rightarrow \mathbb{Z}^{+} \cup\{0\}$ defined by $\lambda_{1}(i)=1$, for all $i \in[m]$, is the optimal edge-discrimiator for $\mathcal{H}_{1}$ and $\omega_{0}\left(\mathcal{H}_{1}\right)=\omega_{\lambda_{1}}(\mathcal{V})=m$ attains the lower bound. 
Remark 3. A hypergraph is called $d$-regular if every vertex is in $d$ hyperedges. Let $\mathcal{H}=(\mathcal{V}, \mathscr{E})$ be a $d$-regular hypergraph with $|\mathscr{E}|=m$. Then any edge discriminator $\lambda$ on $\mathcal{H}$ satisfies $\omega_{\lambda}(\mathcal{V})=\sum_{v \in \mathcal{V}} \lambda(v)=\frac{1}{d} \sum_{E \in \mathscr{E}} \omega_{\lambda}(E) \geqslant \frac{m(m+1)}{2 d}=O\left(m^{2} / d\right)$. If $d=O(1)$, that is, the hypergraph is sparse, this lower bound has the same order of magnitude as the $O\left(m^{2}\right)$ upper bound proved in Theorem 1.

\section{Non-Attainable Optimal Weights: Proof of Theo- rem 3}

We have shown that an optimal edge-discriminator on a hypergraph with $m$ hyperedges can have weight at most $m(m+1) / 2$ and this is attained when the $m$ hyperedges are mutually disjoint. Moreover, there exists a hypergraph for which the weight of the optimal edge-discriminator is $m$, as demonstrated in Proposition 6. This raises the question that whether all weights between $m$ and $m(m+1) / 2$ are attainable. More formally, we are interested in knowing that given any integer $w \in[m, m(m+1) / 2]$, whether there exists a hypergraph $\mathcal{H}(m, w)$ on $m$ edges such that $w$ is the weight of the optimal edgediscriminator on $\mathcal{H}(m, w)$. We say that $w$ is attainable if there exists a hypergraph $\mathcal{H}$ on $m$ edges such that the weight of the optimal edge-discriminator on $\mathcal{H}$ is $w$. An integer $w \in[m, m(m+1) / 2]$ is said to be non-attainable if it is not attainable. In this section we prove Theorem 3 which shows that for all $m \geqslant 3$, the weight $m(m+1) / 2-1$ is non-attainable.

\subsection{Proof of Theorem 3}

For $m=3$, the result can be proved easily by considering the different possible distinct hypergraphs on 3 edges.

Now, fix an integer $m \geqslant 4$. We prove the theorem by contradiction. Assume that there exists a hypergraph $\mathcal{H}_{O}=(\mathcal{V}, \mathscr{E})$, with $|\mathcal{V}|=n$ and $|\mathscr{E}|=m$, such that $\omega_{0}\left(\mathcal{H}_{O}\right)=$ $m(m+1) / 2-1$. Fix any ordering $\nu$ on $\mathcal{V}$. From the proof of Theorem 1 we get an edge discriminator $\lambda^{\prime}$ for $\mathcal{H}_{O}$, such that $\omega_{\lambda^{\prime}}(\mathcal{V}) \geqslant \frac{m(m+1)}{2}-1$.

We now have the following observation:

Observation 7. Under the above assumption, for any fixed ordering $\nu$ on $\mathcal{V}$, either one of the following statements must be true:

(i) There exists $j \in[n]$ such that $\pi\left(\nu_{j}\right)=2$ and $\pi\left(\nu_{k}\right) \leqslant 1$ for all $k \in[n] \backslash\{j\}$.

(ii) $\pi\left(\nu_{k}\right) \leqslant 1$ for all $k \in[n]$.

Proof. Suppose we have an ordering $\nu$ such that $\pi\left(\nu_{j}\right) \geqslant 3$ for some $j$. Without loss of generality, we assume that $j=n$ because otherwise we can work with a new ordering $\nu^{\prime}$ such that $\nu_{j}=\nu_{n}^{\prime}$.

Let $\nu_{n} \in E_{x} \cap E_{y} \cap E_{z}$. We start with the function $\kappa(v)=\delta_{\nu_{n}}$ which takes the value 1 at $\nu_{n}$ and 0 everywhere else. From Lemma 1 we get an edge-discriminator $\lambda_{\kappa, \nu}$ such that 
$\sum_{k=1}^{n} \lambda_{\kappa, \nu}\left(\nu_{k}\right) \leqslant m(m+1) / 2-2$, which contradicts the hypothesis that the optimal edge discriminator has weight $m(m+1) / 2-1$.

Now suppose there are two numbers $i$ and $j$ such that both $\pi\left(\nu_{i}\right)$ and $\pi\left(\nu_{j}\right)$ are equal to 2. Then applying the same argument as above and starting with the function $\lambda=\delta_{\nu_{i}}+\delta_{\nu_{j}}$ and using Lemma 1 we get an edge-discriminator $\lambda_{\kappa, \nu}$ such that $\sum_{k=1}^{n} \lambda_{\kappa, \nu}\left(\nu_{k}\right) \leqslant m(m+$ 1) $/ 2-2$, which gives us a contradiction.

Using the above observation we now formulate the following important lemma:

Lemma 2. No vertex in $\mathcal{V}$ can be incident on more than 2 hyperedges in $\mathscr{E}$.

Proof. If possible, suppose that there exists a vertex $v_{o} \in \mathcal{V}$ such that $v_{o}$ is incident on $\ell(\geqslant 3)$ hyperedges in $\mathscr{E}$. Define the ordering $\nu^{\prime}:[n] \rightarrow \mathcal{V}$, where $|\mathcal{V}|=n$, such that $\nu_{n}^{\prime}:=\nu^{\prime}(n)=v_{o}$. Therefore, $v_{o}$ must be the maximal vertex of all the $\ell$ hyperedges incident on it, that is, $\pi\left(\nu_{n}^{\prime}\right)=\ell \geqslant 3$. This contradicts Observation 7 and the proof of the lemma follows.

Next, suppose that the second possibility in Observation 7 holds for some ordering $\nu$, that is, $\pi\left(\nu_{k}\right)=1$ for all $k \in[n]$. Clearly, $\mathcal{H}_{O}$ cannot be the hypergraph in which all the $m$ are hyperedges disjoint. Therefore, we may assume that at least a pair of hyperedges intersect. Now, similar to the proof of Lemma 2, we can define a new order $\nu^{\prime}$ on the vertices such that $\nu_{n}^{\prime}:=\nu^{\prime}(n) \geqslant 2$, and the problem reduces to the first possibility of Observation 7 with respect to the ordering $\nu^{\prime}$.

Therefore, it suffices to consider the first possibility in Observation 7 , that is, there exists $j \in[n]$ such that $\pi\left(\nu_{j}\right)=2$ and $\pi\left(\nu_{k}\right)=1$ for all $k \in[n]$ and $k \neq j$. Let $F$ and $G$ be the two hyperedges having $\nu\left(i_{j}\right)$ as the maximal vertex. We now have the following lemma:

Lemma 3. For any two hyperedges $A, B \in \mathscr{E} \backslash\{F, G\}, A \cap B=\emptyset$.

Proof. Suppose there exists $v_{o} \in \mathcal{V}$ such that $v_{o} \in A \cap B$. Now, from Lemma 2, $v_{o} \notin F \cup G$. Similarly, as $\nu_{j} \in F \cap G$ we have $\nu_{j} \notin A \cup B$.

Let us consider a new ordering $\nu^{\prime}$ on $\mathcal{V}$ such that $\nu_{n-1}^{\prime}:=\nu^{\prime}(n-1)=\nu_{j}$ and $\nu_{n}^{\prime}:=$ $\nu^{\prime}(n)=v_{o}$. Therefore, $\nu^{\prime}(A)=\nu^{\prime}(B)=v_{o}$, and so $\pi\left(\nu_{n}^{\prime}\right) \geqslant 2$. Moreover, as $v_{o} \notin F \cup G$ and $\nu_{j} \in F \cap G, \nu^{\prime}(F)=\nu^{\prime}(G)=\nu_{j}$. This means that $\pi\left(\nu_{n-1}^{\prime}\right) \geqslant 2$. This contradicts Observation 7 and the result follows.

The above lemma helps us to deduce a necessary configuration of the hypergraph $\mathcal{H}_{O}$. This can be visualized by Figure 1 and is summarized in the following lemma, the proof of which is immediate from Lemma 2 and Lemma 3.

Lemma 4. The set of hyperedges in $\mathscr{E} \backslash\{F, G\}$ can be partitioned into two disjoint sets $\mathcal{F}_{A}=\left\{A_{1}, A_{2}, \ldots, A_{s}\right\}$ and $\mathcal{F}_{B}=\left\{B_{1}, B_{2}, \ldots, B_{t}\right\}$, with $s+t=m-2$, such that:

(i) $A_{i} \cap A_{j}=\emptyset$ and $A_{i} \cap(F \cup G)=\emptyset$ for distinct indices $i, j \in[s]$,

(ii) $B_{i} \cap B_{j}=\emptyset, B_{i} \cap(F \cup G) \neq \emptyset$, and $B_{i} \cap(F \cap G)=\emptyset$ for distinct indices $i, j \in[t]$. 

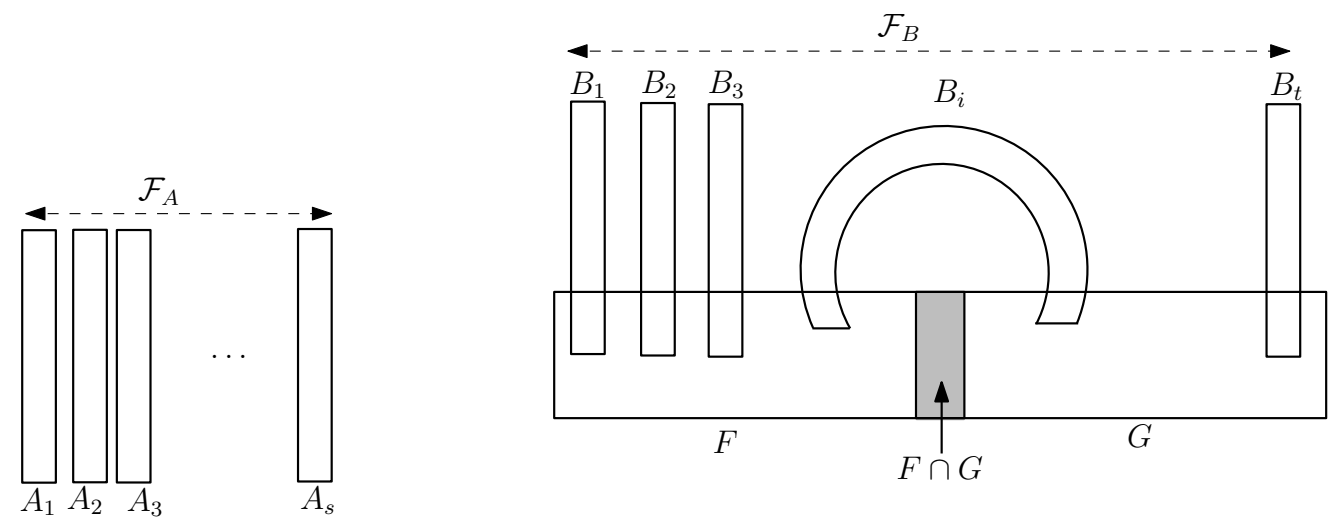

Figure 1: Illustration for the proof of Theorem 3.

Using properties of this special configuration, we now construct an edge-discriminator $\lambda_{1}$ on $\mathcal{H}_{O}$, such that, $\omega_{\lambda_{1}}\left(\mathcal{H}_{O}\right)<m(m+1) / 2-1$.

Case $1 s=m-2$. Then $\left|\mathcal{F}_{B}\right|=0$ and $F$ and $G$ are the only two intersecting hyperedges in $\mathcal{H}_{O}$. We define $\lambda_{1}: \mathcal{V} \rightarrow \mathbb{Z}^{+} \cup\{0\}$ as follows:

$$
\begin{array}{ll}
\lambda_{1}\left(\nu\left(A_{i}\right)\right)=i, & \text { for } i \in[s] ; \\
\lambda_{1}(\nu(F \cap G))=m-1 ; & \\
\lambda_{1}(\nu(F \Delta G))=1 ; & \\
\lambda_{1}(x)=0, & \text { otherwise. }
\end{array}
$$

It is clear that $\lambda_{1}$ is an edge-discriminator on $\mathcal{H}_{O}$, and

$$
\begin{aligned}
\omega_{\lambda_{1}}\left(\mathcal{H}_{O}\right)=\sum_{v \in \mathcal{V}} \lambda_{1}(v) & =\sum_{i=1}^{m-2} \omega_{\lambda_{1}}\left(A_{i}\right)+\omega_{\lambda_{1}}(F)+\omega_{\lambda_{1}}(G)-\omega_{\lambda_{1}}(F \Delta G) \\
& =\frac{m(m-1)}{2}-1<\frac{m(m+1)}{2}-1 .
\end{aligned}
$$

Case $2 s<m-2$. In this case there are $m-s-2$ hyperedges that intersect with $F \Delta G$.

We define $\lambda_{1}^{\prime}: \mathcal{V} \rightarrow \mathbb{Z}^{+} \cup\{0\}$ as follows:

$$
\begin{array}{ll}
\lambda_{1}^{\prime}\left(\nu\left(A_{i}\right)\right)=i, & \text { for } i \in[s] ; \\
\lambda_{1}^{\prime}\left(\nu\left(B_{i} \cap(F \Delta G)\right)\right)=i+s ; & \text { for } i \in[t-1] \\
\lambda_{1}^{\prime}(x)=0, & \text { otherwise. }
\end{array}
$$

Now, we look at

$$
q=\left|\sum_{v \in F} \lambda_{1}^{\prime}(v)-\sum_{v \in G} \lambda_{1}^{\prime}(v)\right| .
$$


If $q=m-1$ then we define $\lambda_{1}: \mathcal{V} \rightarrow \mathbb{Z}^{+} \cup\{0\}$ as

$$
\begin{array}{ll}
\lambda_{1}\left(\nu\left(A_{i}\right)\right)=i, & \text { for } i \in[s] ; \\
\lambda_{1}\left(\nu\left(B_{i} \cap(F \Delta G)\right)\right)=i+s, & \text { for } i \in[t-1] ; \\
\lambda_{1}\left(\nu\left(B_{t} \cap(F \Delta G)\right)\right)=m-2 ; & \\
\lambda_{1}(\nu(F \cap G))=m-1 ; & \\
\lambda_{1}(x)=0, & \text { otherwise. }
\end{array}
$$

If $q \neq m-1$ then we define

$$
\begin{array}{ll}
\lambda_{1}\left(\nu\left(A_{i}\right)\right)=i, & \text { for } i \in[s] ; \\
\lambda_{1}\left(\nu\left(B_{i} \cap(F \Delta G)\right)\right)=i+s, & \text { for } i \in[t-1] ; \\
\lambda_{1}\left(\nu\left(B_{t} \cap(F \Delta G)\right)\right)=m-1 ; & \\
\lambda_{1}(\nu(F \cap G))=m ; & \\
\lambda_{1}(x)=0, & \text { otherwise. }
\end{array}
$$

It is again easy to see that $\lambda_{1}$ is an edge-discriminator on $\mathcal{H}_{O}$ and as $m>3$,

$$
\sum_{v \in \mathcal{V}} \lambda_{1}(v) \leqslant \frac{m(m-1)}{2}+2<\frac{m(m+1)}{2}-1 .
$$

This contradicts our assumption that $\omega_{0}\left(\mathcal{H}_{O}\right)=m(m+1) / 2-1$ and the proof of Theorem 3 follows.

\section{Geometric Set Discrimination and Potential Ap- plications}

In this section we show how hypergraph edge-discriminators can be used to differentiate a collection of regions in $\mathbb{R}^{d}$. Consider a finite collection of regions $\mathcal{R}=\left\{R_{1}, R_{2}, \ldots, R_{m}\right\}$ in $\mathbb{R}^{d}$, where a region is a subset of $\mathbb{R}^{d}$. Given any $m$-tuple $\left(\epsilon_{1}, \epsilon_{2}, \ldots, \epsilon_{m}\right) \in\{0,1\}^{m}$, define $\mathcal{R}\left(\epsilon_{1}, \epsilon_{2}, \ldots, \epsilon_{m}\right)=\bigcap_{i=1}^{m} R_{i}^{\epsilon_{i}}$, where $R_{i}^{0}=R_{i}$ and $R_{i}^{1}=\mathbb{R}^{d} \backslash R_{i}$, for $i \in[m]$. Also for $i \in[m]$, define $E_{i}=\bigcup_{\left(\epsilon_{1}, \epsilon_{2}, \ldots, \epsilon_{m}\right) \in A_{i}} \mathcal{R}\left(\epsilon_{1}, \epsilon_{2}, \ldots, \epsilon_{m}\right)$, where $A_{i}=\left\{\left(\epsilon_{1}, \epsilon_{2}, \ldots, \epsilon_{m}\right) \in\right.$ $\left.\{0,1\}^{n}: \epsilon_{i}=0\right\}$. The geometric hypergraph generated by $\mathcal{R}$, to be denoted by $\mathcal{H}(\mathcal{R})$, is the hypergraph $\left(\mathcal{V}_{\mathcal{R}}, \mathscr{E}_{\mathcal{R}}\right)$, where $\mathcal{V}_{\mathcal{R}}=\left\{\mathcal{R}\left(\epsilon_{1}, \epsilon_{2}, \ldots, \epsilon_{m}\right):\left(\epsilon_{1}, \epsilon_{2}, \ldots, \epsilon_{m}\right) \in\{0,1\}^{n}\right\}$ and $\mathscr{E}_{\mathcal{R}}=\left\{E_{1}, E_{2}, \ldots, E_{m}\right\}$

An edge-discriminator for the geometric hypergraph $\mathcal{H}(\mathcal{R})$ is a finite set $M \subset \mathbb{R}^{d}$ such that $\left|R_{i} \cap M\right|>0$, for $i \in[m]$, and $\left|R_{i} \cap M\right| \neq\left|R_{j} \cap M\right|$, for all $i \neq j \in[m]$. The set $M$ is called the geometric discriminator for $\mathcal{R}$. The optimal edge-discriminator on $\mathcal{H}(\mathcal{R})$ is the geometric discriminator of the least cardinality, and will be called the optimal geometric discriminator of $\mathcal{R}$. The problem of finding the optimal geometric discriminator for a geometric hypergraph, generated by a finite collection of regions in $\mathbb{R}^{d}$, will be called the Geometric Set Discrimination Problem.

Geometric set discrimination seems to be an interesting computational geometry problem, which includes devising efficient algorithms or proving hardness results, particularly 
when the regions consist of intervals in $\mathbb{R}^{1}$, or rectangles or circles in $\mathbb{R}^{2}$. These algorithmic questions are left for future research. However, we shall discuss three simple examples of geometric set discrimination which will provide instructive insights into the properties of edge-discriminators and corroborate some of our earlier results.

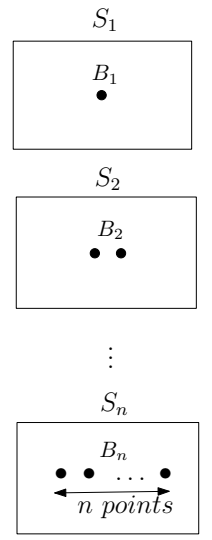

(a)

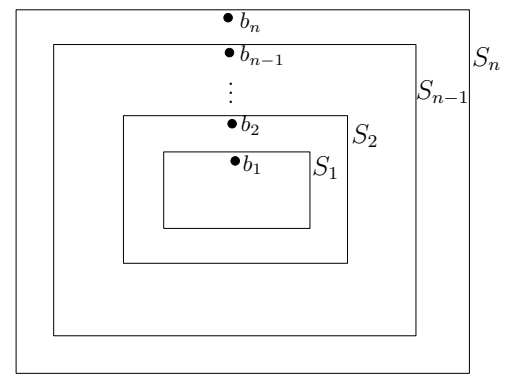

(b)

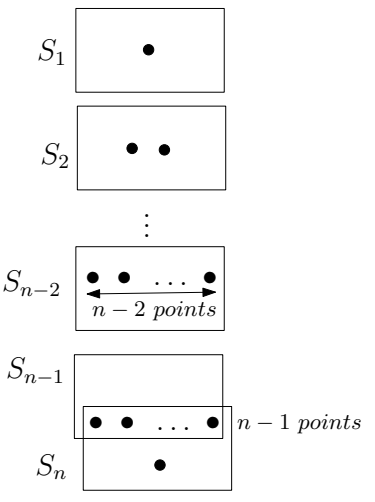

(c)

Figure 2: Examples of geometric set discrimination

Example 1 We have shown that the upper bound on the weight of an edge-discriminator proved in Theorem 1 is attained if and only if the hypergraph has $m$ disjoint edges. The geometric hypergraph generated by the set $\mathcal{S}=\left\{S_{1}, S_{2}, \ldots, S_{m}\right\}$ of mutually disjoint axis-aligned squares (Figure $2(\mathrm{a})$ ) is such an example. Let $B_{i} \subset \mathbb{R}^{2}$ be any set of distinct $i$ points in the interior of $S_{i}$. It is trivial to see that the set $B=\left\{B_{1}, B_{2}, \ldots, B_{m}\right\}$ is the optimum geometric discriminator of $\mathcal{S}$ and $\omega_{0}\left(\mathcal{H}_{\mathcal{S}}\right)=$ $\frac{m(m+1)}{2}$.

Example 2 Consider the set $\mathcal{S}=\left\{S_{1}, S_{2}, \ldots, S_{m}\right\}$ of axis-aligned squares such that $S_{i} \subset$ $S_{i+1}$ for all $i \geqslant 1$. Let $B=\left\{b_{1}, b_{2}, \ldots, b_{m}\right\}$, where $b_{i} \in S_{i} \backslash \bigcup_{j=1}^{i-1} S_{j}$ (Figure 2(b)). Clearly, $B$ is the optimum edge-discriminator of the geometric hypergraph $\mathcal{H}_{\mathcal{S}}$. Since $|B|=m$, the optimum-weight of the edge-discriminator of $\mathcal{H}_{\mathcal{S}}$ attains the lower bound in Proposition 6.

Example 3 Consider the set $\mathcal{S}=\left\{S_{1}, S_{2}, \ldots, S_{m}\right\}$ of axis-aligned squares, such that $S_{i} \cap$ $S_{j}=\emptyset$ for all $i \neq j \in[m-1]$, and $S_{i} \cap S_{m}=\emptyset$ for all $i \in[m-2]$, and $S_{m-1} \cap S_{m} \neq \emptyset$ (see Figure 2(c)). Let $B_{i}$ be any set of $i$ points in the interior of $S_{i}$, for $i \in[m-2]$. Let $B_{m-1}$ be any set of $m-1$ points in the interior of $S_{m-1} \cap S_{m}$ and $b_{m}$ is any point in the interior of $S_{m} \backslash S_{m-1}$. It is easy to see that set $B=\left\{B_{1}, B_{2}, \ldots, B_{m-1}, b_{m}\right\}$ is the optimum geometric discriminator of $\mathcal{S}$, with $\omega_{0}\left(\mathcal{H}_{\mathcal{S}}\right)=m(m-1) / 2+1$. Note that in this example the $m$ hyperedges are almost disjoint, but the weight of the optimal edge-discriminator is $\frac{m(m-1)}{2}+1=\frac{m(m+1)}{2}-(m-1)$. In fact, this example leads us to conjecture that all integer values in $\mathcal{N}:=\left[\frac{m(m-1)}{2}+2, \frac{m(m+1)}{2}-1\right]$ are non-attainable. In Theorem 3 we only show that $\frac{m(m+1)}{2}-1$ is non-attainable. 
Geometric set discrimination problems may find many potential applications to image indexing in a large database [3, 4], where the emphasis is specially given on deciding whether a particular image exists in the database, rather than on finding the similarity matches of the given image. A novel method for archival image indexing using only the number of connected components, the number of holes, or the Euler number of an image was reported by Biswas et al. [4]. A connected component of a digital binary image is a subset of maximal size such that any two of its pixels can be joined by a connected sequence of pixels assuming 8-connectivity, lying entirely in the subset. A hole in a digital image is a region of the background, which is a connected component in 4-connectivity and is completely enclosed by the object. The Euler number of an image is defined as the number of connected components minus the number of holes in the image. If $C$ and $H$ denote the number of connected components and the number of holes in a digital image, respectively, then its Euler number $E=C-H[3,21,32]$. The ordered pair $(C, H)$ is called the Euler pair of a digital image. It is apparent that two or more images may have the same value of the Euler pair, and hence this feature alone often cannot uniquely characterize an image in a large database. One way to disambiguate the features is to deploy a mask image [4] as follows: We assume that each image is given as a $\left(k_{1} \times k_{2}\right)$ binary pixel matrix. Let us consider $m$ images $I_{1}, I_{2}, \ldots, I_{m}$, each having the same Euler pair. In order to discriminate them, another binary image $M$, called the mask, is to be constructed such that the Euler pair of the $m$ images $I_{1} \odot M, I_{2} \odot M, \ldots, I_{m} \odot M$ are mutually distinct, where $\odot$ denotes bitwise Boolean operation, like XOR or AND between the corresponding bits of the two pixel matrices.

As it turns out, finding a simple mask of a given set of images is a challenging problem. Biswas et al. [4] provided an iterative heuristic using a few synthetic pseudo-random masks. We now show that finding a mask for a set of images can be modeled as an instance of the hypergraph edge-discrimination for a collection of geometric regions in $\mathbb{R}^{2}$. Consider the images $I_{1}, I_{2}, \ldots, I_{m}$, superimposed on each other in the same frame, as subsets of $\mathbb{R}^{2}$. Suppose $M$ is a geometric discriminator for this collection of regions. Then the binary image corresponding to $M$ is a mask for the set of images under the bitwise Boolean AND operation. The process is illustrated with four binary images in Figure $3 .{ }^{1}$ The mask corresponding to the optimal geometric discriminator is the simplest in the sense that the pixel matrix has the least number of ones.

\section{Conclusions}

In this paper we introduce the notion of hypergraph edge-discrimination and study its properties. We show that given any hypergraph $\mathcal{H}=(\mathcal{V}, \mathscr{E})$, with $|\mathcal{V}|=n$ and $|\mathscr{E}|=m$, $\omega_{0}(\mathcal{H}) \leqslant m(m+1) / 2$, and the equality holds if and only if the elements of $\mathscr{E}$ are mutually

\footnotetext{
${ }^{1}$ As binary images are actually subsets of the discrete space $\mathbb{Z}^{2}$, the mask $M$ should be a subset of $\mathbb{Z}^{2}$. As a result, several technical difficulties may arise while trying to obtain a geometric discriminator for a set of binary images containing holes. These problems need to be handled separately and they are not of interest to this paper. Here we discuss the unique indexing problem as a motivating application for the edge-discrimination problem on hypergraphs.
} 


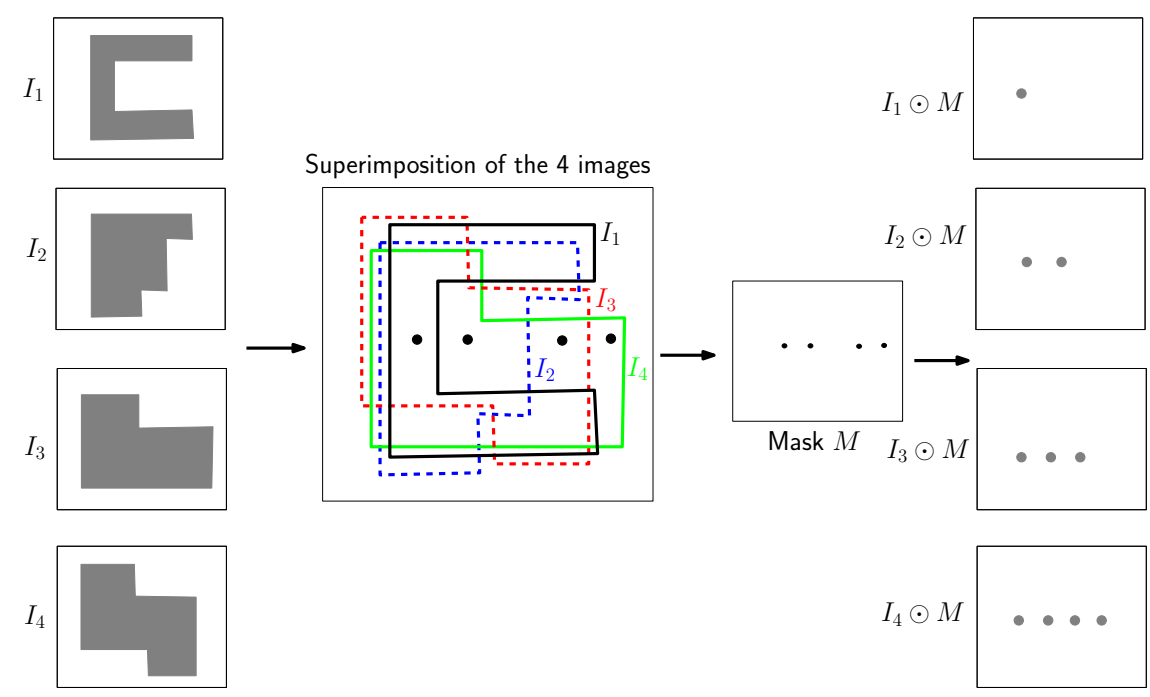

Figure 3: Unique image-indexing by geometric-set discrimination.

disjoint. For $r$-uniform hypergraphs, using properties of $B_{h}$-sets, we prove that $\omega_{0}(\mathcal{H}) \leqslant$ $n^{r+1}+o\left(n^{r+1}\right)$, and the bound is attained by the complete $r$-uniform hypergraph up to a constant factor.

We also considered the question of attainability of weights: Given any integer $w \in$ $[m, m(m+1) / 2]$, whether there exists a hypergraph $\mathcal{H}(m, w)$ with $m$ hyperedges such that the weight of the optimal edge-discriminator on $\mathcal{H}(m, w)$ is $w$. We answer this question in the negative by proving that there exists no hypergraph on $m(\geqslant 3)$ hyperedges such that the weight of the optimal edge-discriminator is $m(m+1) / 2-1$. The problem of characterizing non-attainable weights remains open and might have interesting consequences.

Also, as mentioned in the previous section, another problem for future research is the algorithmic study of the geometric set-discrimination problem.

\section{Acknowledgements}

The authors thank the anonymous referees for valuable comments which improved the presentation of the paper.

\section{References}

[1] I. Aliev, Siegel's lemma and sum-distinct sets, Discrete and Computational Geometry, Vol. 39 (1-3), 59-66, 2008.

[2] B. B. Bhattacharya, S. Das, S. Ganguly, Minimum-weight edge discriminator in hypergraphs, arXiv:1210.4668v2, 2012. 
[3] A. Bishnu, B. B. Bhattacharya, M. Kundu, C. A. Murthy, T. Acharya, Euler vector for search and retrieval of gray-tone images, IEEE Transactions on Systems, Man, and Cybernetics, Vol. 35, 801-811, 2005.

[4] A. Biswas, P. Bhowmick, B. B. Bhattacharya, Archival image indexing with connectivity features using randomized masks, Applied Soft Computing, Vol. 8 (4), 1625-1636, 2008.

[5] T. Bohman, A construction for sets of integers with distinct subset sums, Electronic Journal of Combinatorics Vol. 5, \#R3, 1-14, 1998.

[6] B. Bollobás, O. Pikhurko, Integer sets with prescribed pairwise differences being distinct, European Journal of Combinatorics, Vol. 26 (5), 607-616, 2005.

[7] R. C. Bose, S. Chowla, Theorems in the additive theory of numbers, Comment. Math. Helv., Vol. 37, 141-147, 1962/1963.

[8] G. Chartrand, M. S. Jacobsen, J. Lehel, O. R. Oellermann, S. Ruiz, F. Saba, Irregular networks, Congressus Numerantium, Vol. 64, 187-192, 1988.

[9] J. Cilleruelo, Sidon sets in $\mathbb{N}^{d}$, Journal of Combinatorial Theory, Series A. Vol. 117 (7), 857-871, 2010.

[10] J. Cilleruelo, New upper bounds for $B_{h}$ sequences, Advances in Mathematics, Vol. 159 (1), 2001.

[11] J. Cilleruelo, J. Jimenez, $B_{h}[g]$ sequences, Mathematik, Vol. 47 (1-2), 2000.

[12] J. H. Conway, R. K. Guy, Sets of natural numbers with distinct subset sums, Notices, American Mathematical Society Vol. 15, 345, 1968.

[13] G. Ebert, J. Hammenter, F. Lazebnik, A. Woldar, Irregularity strengths for certain graphs, Congressus Numerantium, Vol. 71, 39-52, 1990.

[14] N. Elkies, An improved lower bound on the greatest element of a sum-distinct set of fixed order, Journal of Combinatorial Theory, Series A, Vol. 41, 89-94, 1986.

[15] P. Erdős, Problems and results from additive number theory, Colloq. Théorie des nombres, Bruxells, 127-137, 1955.

[16] P. Erdős, P. Turán, On a problem of Sidon in additive number theory and on some related problems, Journal of the London Mathematical Society, Vol. 16, 212-215, 1941. Addendum, Vol. 19, 208, 1944.

[17] R. J. Faudree, M. S. Jacobson, J. Lehel, R. H. Schelp, Irregular networks, regular graphs and integer matrices with distinct row and column sums, Discrete Mathematics, Vol. 76, 223-240, 1989.

[18] R. J. Faudree, J. Lehel, Bound on the irregularity strength of regular graphs, Colloq. Math. Soc. János Bolyai, 52, Combinatorics, Eger. North Holland, Amsterdam, 247-256, 1987.

[19] A. Frieze, R. Gould., M. Karoński, F. Pfender, On graph irregulaity strength, Journal of Graph Theory, Vol. 41, 120-137, 2002.

[20] J. A. Gallian, A dynamic survey of graph labeling, Electronic Journal of Combinatorics, 17, \#DS6, 2010.

[21] R. C. Gonzalez and R. E. Woods, Digital Image Processing, Addison-Wesley, California, USA, 1993. 
[22] R. K. Guy, Sets of integers whose subsets have distinct sums, Theory and Practice of Combinatorics, edited by A. Rosa, G. Sabidussi, and J. Turgeon, Annals of Discrete Mathematics, Vol. 12, 141-154, North-Holland, Amsterdam, 1982.

[23] A. Gyárfás, M. Jacobson, L. Kinch, J. Lehel, R. Schelp, Irregularity strength of uniform hypergraphs, J. Comb. Methods Comb. Comput., Vol. 11, 161-172, 1992.

[24] H. Halberstam, K. F. Roth, Sequences, Springer-Verlag, New York, 1983.

[25] X. D. Jia, On finite Sidon sequences, Journal of Number Theory, Vol. 49, 246-249, 1994.

[26] M. Kalkowski, M. Karonski, F. Pfender, A new upper bound for the irregularity strength of graphs, SIAM Journal on Discrete Mathematics, Vol. 25(3), 1319-1321, 2011.

[27] J. Lehel, Facts and quests on degree irregular assignments, 6th International Conference on Graph Theory, Combinatorics, and Applications, (Kalamazoo, MI, 1988), WileyInterscience Publications, Vol. 2, 765-782, 1991.

[28] B. Lindström, An inequality for $B_{2}$-sequences, Journal of Combinatorial Theory, Vol. 6, 211-212, 1969.

[29] W. F. Lunnon, Integers sets with distinct subset sums, Mathematics of Computation, Vol. 50, 297-320, 1988.

[30] T. Nierhoff, A tight bound on the irregularity strength of graphs, SIAM Journal on Discrete Mathematics, Vol. 13, 313-323, 2000.

[31] K. O'Bryant, A complete annotated bibliography of work related to Sidon sequences, Electronic Journal of Combinatorics, 11, 2004.

[32] W. Pratt, Digital Image Processing, John Wiley and Sons, 1978.

[33] J. Przybyło, Irregularity strength of regular graphs, Electronic Journal of Combinatorics, Vol. 15, (1), \#R82 10, 2008.

[34] J. Przybyło, Linear bound for on the irregularity strength and the total vertex irregularity strength of graphs, SIAM Journal on Discrete Mathematics, Vol. 23 (1), 511-516, 2009.

[35] J. Singer, A theorem in finite projective geometry and some applications to number theory, Transactions of the American Mathematical Society, Vol. 43, 377-385, 1938.

[36] D. R. Wood, On vertex-magic and edge-magic total injections of graphs, Australasian Journal of Combinatorics, Vol. 26, 49-63, 2002. 